\title{
El Taller de Pintura de la Quinta Heeren (1906-1916). Prácticas de pintura al natural en manos femeninas ${ }^{i}$
}

Fernando Villegas Torres

Pontificia Universidad Católica del Perú

El presente ensayo propone un estudio del primer taller de pintura al aire libre en Lima que tuvo entre sus integrantes a pintoras mujeres. En este espacio, si bien se siguieron las enseñanzas del maestro Teófilo Castillo, pronto las pintoras manifestaron sus lenguajes artísticos en la incursión del retrato simbolista. La introducción del paisajismo de carácter naturalista reveló un primer avance hacia el desarrollo de la plástica peruana.

Pintura al aire libre, Teófilo Castillo, María Angélica Quincot, Perú Antiguo, paisajismo, taller de pintura, educación artística, pintoras.

This article investigates the first outdoor studio of plein air painting in Lima, Peru, which had between its participating members a large number of women painters. At first these students followed quite rigidly the instructions of their teacher, the painter Teofilo Castillo, but gradually they developed their own artistic modes of expression, with the creation of Symbolist portraits. The introduction of plein air landscape painting in Lima was a first step in the development of modern Peruvian painting.

Plein air painting, Teófilo Castillo, María Angélica Quincot, Ancient Peru, landscape painting, outdoor studio, artistic education, women painters.

El primer taller de pintura al aire libre tuvo un alumnado mayoritariamente femenino ${ }^{2}$ que ha sido poco estudiado. El objetivo del presente artículo será ver sus alcances e implicancias en el contexto local limeño. Para la ciudad, caracterizada por el predominio del género retrato y por un deficiente marco institucional en la enseñanza del arte, el taller de pintura al aire libre significó el primer avance hacia la modernidad en el arte peruano con la introducción del paisajismo de carácter naturalista. La academia fue instituida por el pintor y crítico de arte Teófilo Castillo, que se estableció en la letra $L$ de la Quinta Heeren, en el actual distrito de Barrios Altos de la ciudad de Lima.

Los inicios del taller de pintura

Era 1906 y Teófilo Castillo, el artista peruano llegado recientemente de Buenos Aires, puso un anuncio -que no especificaba la edad ni el género del público al que iba dirigido- en el que ofrecía clases de pintura. La convocatoria dio como resultado que señoritas de buena

1 El presente artículo pertenece a una parte del segundo capítulo de mi tesis doctoral en Historia del Arte: Vínculos artísticos entre España y Perú (1892-1929). Elementos para la construcción del imaginario nacional peruano por la Universidad Complutense de Madrid, ver: (Villegas, 2013). Defendida ante tribunal el 04 de julio del 2013 donde obtuvo la máxima calificación.

2 Entendemos para los estudios de género lo dicho por Jill K. Conway, Susan C. Bourque y Joan W. Scott que refieren que: aprender sobre las mujeres implica tambien aprender de los hombres. El estudio de genero es una manera de comprender a las mujeres no como un aspecto aislado de la sociedad sino como parte integral de ella (Conway, Bourque y Scott, 1997:11). Esta relación entre las pintoras mujeres y su maestro en la sociedad patriarcal limeña estarán implícitos a lo largo del artículo. 


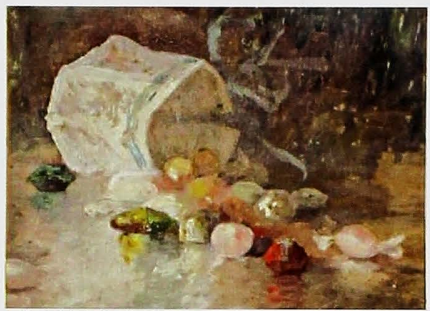

Fig.1.- María Angélica Quíncot, Naturaleza muerta (c.1906-07), óleo sobre lienzo, colección particular.

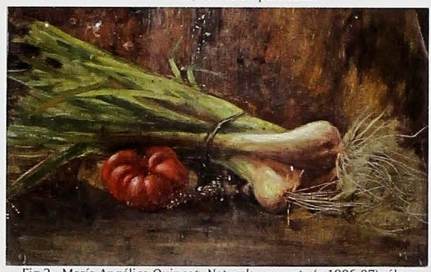

Fig.2.- María Angélica Quincot, Naturaleza muerta (c.1906-07), óleo sobre lienzo, colección particular.

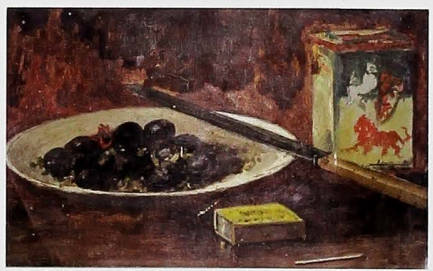

Fig.3.- María Angélica Quincot, Naturaleza muerta (c.1906-07), óleo sobre lienzo, colección particular. familia fueran el público más interesado $^{3}$. Se trataba de jóvenes cultas, vinculadas a sectores profesionales e intelectuales de la alta burguesía limeña ${ }^{4}$, quienes, desde luego, compartían un mismo círculo social ${ }^{5}$.

Para comprender por qué la ciudad de Lima tenía una academia de pintura con un marcado ingrediente femenino es necesario conocer los antecedentes entre las artes y las prácticas femeninas de entonces. Ya en febrero de 1840 , el artista italiano Camilo Domeniconi anunció clases particulares de dibujo y pintura para señoritas en sus propias casas. Por esos mismos años, la italiana Sabina Meucci dio clases de dibujo en un colegio para señoritas junto a su padre. La preferencia del espacio público para los hombres y el privado para las mujeres se mantiene hasta finales del siglo XIX, con la creación de la Academia Concha (1897), donde, a pesar de no estar restringido su ingreso, el horario nocturno imposibilitó la presencia femenina, pues no era apropiado que salieran de noche según las normas sociales de aquella época (Pachas, 2008:43).

De hecho, el espacio donde las mujeres podían estudiar arte se encontraba en el taller particular del artista, tal como sucede en las academias del pintor italiano Leonardo Barbieri, pues ya en la Exposición de 1860 participan algunas damas. Lo mismo sucede, a finales del siglo, en el taller del artista español Ramón Muñiz. También se pueden mencionar las clases impartidas por

3 La participación femenina en el ámbito cultural limeño no sólo se circunscribía a las artes plásticas: en el campo de las letras, las tertulias de la argentina Juana Manuela Gorriti, realizadas a partir de 1875 . establecieron las pautas para el florecimiento de una generación de escritoras, ver: (Denegri, 2004).

4 Entre ellas figuran María Flórez Quintanilla, hija del doctor Ricardo Flórez, diputado por el gobierno de José Pardo; René Palma, hija del escritor Ricardo Palma; Mercedes Dammert, hija de la mecenas a favor de los niños Juana Larco de Dammert; y Julia Codesido, hija del abogado y diplomático peruano Bernardino Codesido Oyague.

5 La amistad de las alumnas del taller de pintura de la Quinta Heeren es corroborada por las opiniones de sus descendientes, que recuerdan su participación en reuniones sociales hasta su madurez, así como los cuadros que se regalaron entre ellas y que conservaron sus respectivas familias. 
Carlos Baca Flor durante su estancia en Lima (1888 -1889) a las señoritas Luisa Gastañeta y Hortensia Cáceres ${ }^{6}$. Otros talleres activos a finales del siglo, ya regidos por pintoras, son los de la italiana Valentina Pagani y las peruanas Rebeca Oquendo y su discípula Rosa Angélica Romero. Esta última impartía lecciones de dibujo a señoritas ("Concurso Concha", 1903:22), a diferencia de Pagani que, en un primer momento, enseñó a particulares y luego exclusivamente a señoritas (Pachas, 2008:45-46). De acuerdo con los planteamientos del taller, los alumnos se formarían en el conocimiento de la naturaleza, pues sólo a través de sus formas podían entender la verdad del arte.

Castillo, que no cree en la virtud paciente y estéril del dibujo enseñado ante el yeso, en salas descoloridas, hace beber á sus discípulos en las fuentes inagotables del natural (...) propicia engendrar en el alma del alumno, la desilusión y el desengaño, ante las dificultades técnicas y sin tener el recurso del color, cuyo empleo estimula al hierofante á proseguir y mejorar en el arte elegido ("El arte en Lima...", 1906:829-830).

Los alumnos del taller seguían la enseñanza del dibujo concreto planteado por el argentino Martín Malharro ${ }^{7}$, frutas y objetos cotidianos ayudarían a los discípulos a entender las particularidades de las formas y las distintas gradaciones de color (Fig.1, Fig.2, Fig.3). El uso de objetos cotidianos constituía un primer paso significativo en la introducción de la pintura moderna en el país. Se rechazó la pintura que buscaba transmitir contenidos grandilocuentes e ideas, es decir, la pintura de historia. Al pintarlos se desechaba toda preocupación por el contenido, cuestión que había caracterizado buena parte de la pintura desde el Renacimiento hasta mediados del siglo XIX. El contenido religioso, literario, político e histórico era una carga para la pintura moderna. Así lo entendió el pintor francés Édouard Manet y su escuela quienes serían los primeros en erradicarlo de su pintura.

El único centro de enseñanza que existía en esos años era la Academia Concha, institución creada por iniciativa de la peruana Adelina Concha en $1897^{\circ}$, donde se impartían cursos de dibujo natural y lineal. El primero, destinado a futuros artistas, se basó en la copia de modelos en yeso, por eso la propuesta de la Quinta Heeren se diferenciaba de las demás academias de dibujo fundadas en el siglo $\mathrm{XIX}^{9}$ por desarrollar no el dibujo sino la pintura del natural en óleo ${ }^{10}$, así seguía más la tradición de las escuelas de pintura fundadas por los pintores locales y extranjeros donde se formaron varios artistas peruanos antes de partir a Europa. El taller fue una innovación en un contexto carente de institucionalidad artística, donde raras veces se realizaba una exposición, y caracterizado por la consiguiente ausencia de un mercado para las obras de arte.

El taller de pintura al aire libre ubicado en la Quinta Heeren tuvo un carácter conservador e innovador a la vez. Funcionó durante diez años, entre 1906 y $1916^{11}$; los grupos de alumnos se reunían dos veces por semana para pintar del natural paisajes y figuras (Castillo, 1918: 1053).

6 Para mayor información sobre las pintoras anteriores al taller en la Quinta Heeren, ver: (Pachas 2008:33-41).

7 En 1919 el propio Castillo afirmó haber estado suscrito a las enseñanzas del pintor argentino Malharro, a quien consideró el creador del dibujo sintético y cuya metodología describió como luminosa, racional y simple (Castillo, 1919e:1010).

8 Sobre la historia de la Academia Concha, ver: (Pachas, 2005).

9 Para un estudio sobre las academias de dibujo en Lima ver: (Majluf, 1993:32-42).

10 Una excepción a lo mencionado es el pintor colombiano Manuel Carbajal quien el 27 de septiembre de 1849 abrió un aula del dibujo en el colegio de Beausejour. Ver: (Majluf, 1993: 36).

11 Considerando el viaje a España del artista, realizado entre 1908 y 1909 , se debería suponer que durante esos años la academia cerró; sin embargo, por el propio pintor se sabe que se mantuvo abierta de manera ininterrumpida durante diez años en la mencionada Quinta Heeren (Castillo, 1918:1053). El tiempo de duración del taller sería reiterado en dos artículos más escritos por el pintor: (Castillo, 1919a:345-347) y (Castillo, 1919d:683-684). 


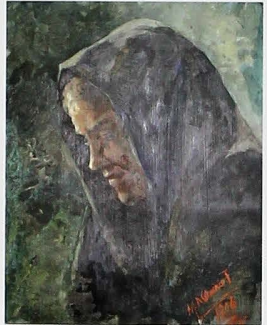

Fig.4.- María Angélica Quincot, Retrato de señora con manta (c. 1906-07), óleo sobre lienzo, colección particular.

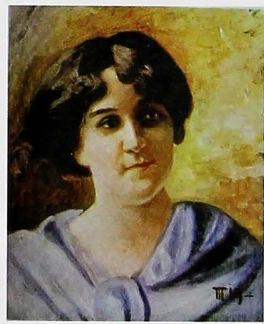

Fig.5.-María Isabel Arenas y Loayza, Retrato de María Isabel Sánchez Concha (Variedades, Lima, No 448,30 XI 1916).

Fue el maestro nacional don Teófilo Castillo, el primero que estableció una escuela al aire libre, haciendo que el grupo de sus discípulas, que no fue muy reducido, le siguiera en sus magníficas lecciones, dictadas en plena naturaleza: en el recinto de alguna arboleda, a la orilla del mar, en un jardín o en cualquier sitio, donde hubiera belleza que impresionara la retina de las discípulas (García, 1925:511) ${ }^{12}$.

Su carácter más convencional y conservador se manifestó en el uso de los modelos, un anciano de largas barbas y dos mujeres, así como la aparente separación de los grupos de alumnos de acuerdo a su género. Este aspecto se evidencia además por los temas representados. Se realizaban retratos de un tercio sin ninguna preocupación por la anatomía humana. El desnudo, como sería propio de una academia oficial, estaba comple-

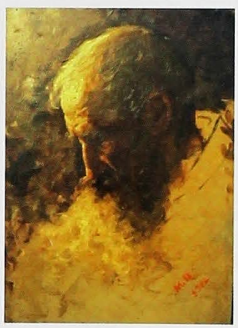

Fig.6.- Mercedes Dammert, Retrato de anciano, (c.1906-07), óleo sobre lienzo, colección particular. tamente ausente en la Quinta Heeren, aunque era común en las academias europeas y constituía el motivo principal por el cual las mujeres no podían acceder a sus clases. El hecho de que el taller tuviera un componente mayoritariamente femenino pudo ser un obstáculo para la realización del desnudo, especialmente el masculino.

En el taller de pintura de la Quinta Heeren, las alumnas pintaban el rostro con modelos colocados de tres cuartos: por lo tanto, existía una preocupación por la expresión de los personajes retratados ${ }^{13}$. En el caso del modelo femenino, en un primer momento se escogió a una dama desconocida con mantilla, tal como se observa en las pinturas de María Angélica Quincot y Mercedes Dammert. La primera modelo, una señora retratada por Quincot que posó entre 1906-1908 (Fig.4), no corresponde a la joven María Isabel Sánchez Concha (Fig.5), modelo entre 1910 y 1916 como demuestran los cuadros de Dammert y Arenas y Loayza y también lo confirma Castillo: En una época, ella fue la modelo favorita de mi academia de pintura en la Quinta Heeren, y fue, durante esas horas de charla íntima y continua, mientras posaba para un grupo distinguido de señoritas pintoras, que yo pude estudiar algo su rico temperamento de artista... (Castillo, 1916d:1280).

12 Una de las primeras investigaciones que evidenció la importancia de los estudios de pintura de la Quinta Heeren como primera institución dedicada a la pintura al aire libre con un marcado componente femenino, fue Elvira García García en La mujer peruana a través de los siglos (1925).

13 Un académico consideraría que los retratos realizados por las alumnas, que seguían los planteamientos de su maestro, no presentan un aspecto acabado, parecen haber sido dejados sin concluir. Sin embargo, se trata de un sintoma de modernidad que develaba el criterio del maestro, que prefería el color y la mancha al acabado perfecto del dibujo. 
En la presentación del taller, Actualidades reveló la separación entre los modelos asignados a los alumnos: un viejo de largas barbas patriarcales para las niñas, y una señora, andaluza ella y de canónica edad, para los hombres ("El arte en Lima...", 1906:829). Sin embargo, hoy se sabe que en el taller los modelos eran compartidos, y ellas podían retratar tanto al anciano (Fig.6) como a la señora. Esto se evidencia en una fotografía incluida en el mismo artículo que muestra a la alumna del taller María Flórez y una modelo con un manto al fondo. La historiadora del arte Lisa Tickner refiere la necesidad de especificar las prácticas particulares del hombre y de la mujer en su producción para examinar a través de ellas la diferencia cultural (Tickner, 2001:253-254). No cabe duda que las obras que realizaron las pintoras de la Quinta Heeren estuvieron delimitadas por su condición femenina, no obstante el hecho de no realizar la figura humana no les imposibilitó ser modernas. Siendo ellas quienes al pintar al aire libre pusieron la pauta de lo considerado moderno en la plástica peruana de principios del siglo $\mathrm{XX}^{14}$.
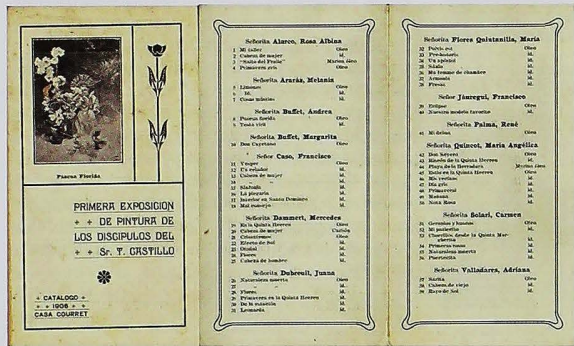

Fig.7.- Catálogo de la primera exposición de pintura de los discípulos del Sr. Teófilo Castillo. 1906, Casa Courret.

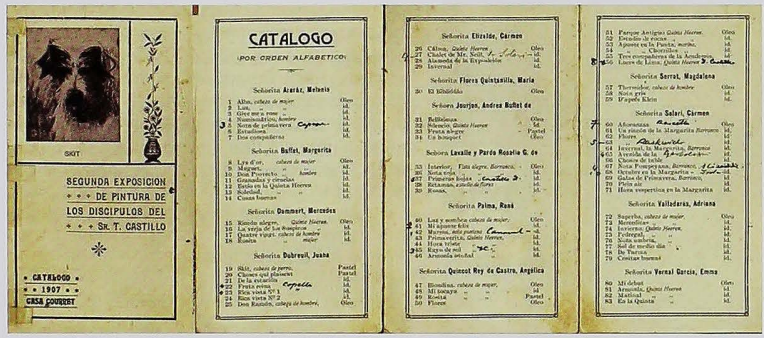

Fig.8.- Catálogo de la segunda exposición de pintura de los discípulos del Sr. Teófilo Castillo, 1907. Casa Courret.

14 No obstante debemos mencionar los iniciales trabajos de José María Eguren quién hizo paisajes siguiendo la enseñanza de Valentina Pagani de Casoratti o las propuestas en el mismo género de Carlos Jiménez y Luis Astete y Concha. Sin embargo no se tiene noticias que estos artistas hayan realizado su obra al natural. Por ello la propuesta del taller diferenciaba la representación del paisaje académico concluido en estudio del pintado al aire libre. 


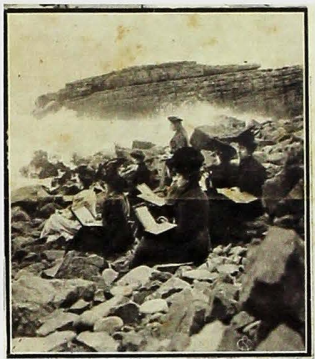

Fig.9 Fotografía de las alumnas de la Quinta Heeren pintando una marina en la playa de la Herradura, Chorrillos, (Actualidades, Lima, Nº176, 11 VIII 1906).

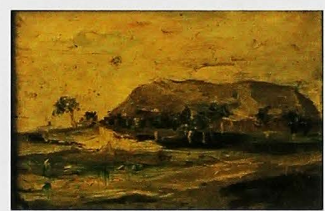

Fig.10 Mercedes Dammert, Estudio de Chorrillos (c.1906-07), óleo sobre lienzo, colección particular.

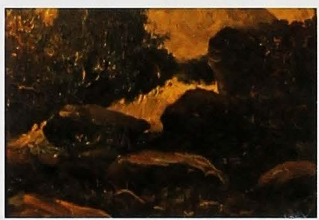

Fig.11 Teófilo Castillo, Estudio de Rocas (1912), óleo sobre cartón, $25 \times 21 \mathrm{~cm}$, Lima, colección particular.

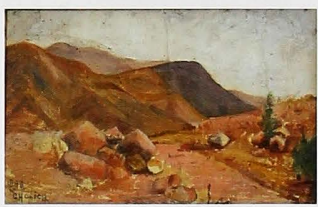

Fig.12 María Angélica Quincot, Estudio de paisaje (1912), óleo sobre lienzo, colección particular.
A finales del año y como culminación del aprendizaje, se realizó en 1906, en la Casa fotográfica Courret, el llamado Salón Castillo o vernissage, una exhibición de los trabajos de sus discípulos, considerada como la primera fiesta de este género que se celebra en esta capital ("Salón Castillo...", 1906:1396). Actualidades cubrió el evento y publicó dos fotografías en las que puede apreciarse que la mayor parte de los alumnos eran mujeres. La prensa de la época informó únicamente sobre los dos primeros salones celebrados; sin embargo se sabe por el propio pintor que éstos continuaron hasta 1912 (Castillo, 1914a:1521).

En las fotografías aparecían los dos únicos alumnos que participaron en la primera exposición de 1906, Francisco Caso y Francisco Jáuregui: al siguiente año se evidenció la participación femenina de manera exclusiva. Ver el catálogo de las dos primeras exposiciones que se reproducen por primera vez en este artículo (Fig.7 y Fig.8) Si comparamos los temas que desarrollaron con los de las alumnas, en realidad se observan prácticas comunes a ambos sexos, algo que podría deberse a las clases conjuntas realizadas en el taller, como estudios del natural, objetos cotidianos y retratos.

Aunque se ha mencionado que el propio Castillo dio indicios de prejuzgar el trabajo de sus alumnas al condicionar el uso de la técnica del pastel a la mano femenina (Pachas, 2008:70-71): (...) el pastel ofrece cierta novedad entre nosotros (...) sin embargo de ser una técnica fácil, bella apropiada para delicadas manos femeniles (Castillo, 1914a:1521). Más adelante definió la técnica del pastel como un trabajo secundario respecto al óleo, y señaló a este último como el mejor camino para desarrollar la pintura. Se hace necesario aclarar que en la primera muestra (1906) de sus discípulas, cincuenta y ocho de los cincuenta y nueve cuadros presentados fueron realizados al óleo, a excepción del carbón Cabeza de mujer de Mercedes Dammert. La introducción del pastel se produjo a partir de la segunda exposición (1907), donde ocho de las ochenta y tres pinturas exhibidas estaban realizadas en esta técnica (Catálogo...., 1907). En los años siguientes, Castillo siguió inculcando en sus alumnas el trabajo al pastel, como lo demuestra la Cabeza de niña de Clotilde Porras de Osma, presentada en el último Salón Castillo (1912) (Castillo, 1914a:1521). Muchos 
de los apuntes en pequeño formato de vistas del natural de paisajes e interiores, tanto de María Angélica Quincot como de René Palma, se realizaron con este procedimiento artístico. Que las alumnas pinten al óleo en la mayoría de cuadros contradice la delimitación del pastel a la mano femenina y por tanto su diletantismo. Todo lo contrario, la práctica del óleo en ellas es evidencia que Castillo buscó la profesionalización artística en el único medio considerado más idóneo para ello.

Pintura al aire libre: jardines, paisajes de la playa y elementos rocosos

Sin lugar a dudas, la mayor contribución del arte moderno, y en particular del Impresionismo, había sido salir del estudio y pintar del natural. Éste fue el signo distintivo del taller de Castillo: sus alumnos salían al aire libre y copiaban la naturaleza como principal requisito de su aprendizaje. Muestra de ello son dos fotografías de la época donde aparecen las jóvenes discípulas pintando en la playa de la Herradura, al sur de Lima, en el distrito de Chorrillos (Fig.9). Estas vistas, junto a las del maestro tomando apuntes del natural y la obra terminada, aparecieron en Actualidades en un artículo que se hacía eco de la academia y de su nueva propuesta. Mercedes Dammert hizo un boceto de la ruta seguida por el maestro y las alumnas camino al mar, desde Lima a Chorrillos (Fig.10), una verdadera excursión para la época. En el apunte se divisaba el Morro Solar rodeado de árboles y terrenos de campos de cultivo, hoy absorbidos por la urbe limeña.

Castillo comenta que después de 1910 también se hicieron excursiones hacia Chosica, un distrito ubicado en el camino a la sierra central de la capital peruana, de tierras montañosas y clima cálido, donde la naturaleza invitaba su representación a través del pincel. En el Estudio de rocas (Fig.11) de 1912, se representa una zona pedregosa junto a una típica caída de agua de este paisaje peruano. A esa misma época corresponde el Estudio de paisaje (Fig.12), pintado por María Angélica Quincot, en el que plasmó una zona de paisaje agreste con cerros, casi desértica, con algunos elementos rocosos en primer plano.

El análisis de estos lugares evidencia la propuesta de Castillo: se trataba de adecuar la percepción visual del estudiante no a la totalidad del paisaje, según el canon occidental europeo, sino privilegiando el primer plano, algún detalle o elemento. Según la opinión del maestro, sólo a partir de esta primera mirada se podía hacer una composición mayor. El mismo procedimiento fue utilizado en el estudio de los Jardines de la Quinta Heeren, fechado en 1906 por Castillo (Fig.13) que guarda sintonía con los

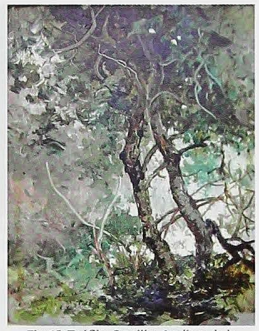

Fig.13 Teófilo Castillo, Jardines de la Quinta Heeren (1906), óleo sobre lienzo. Lima, colección particular.

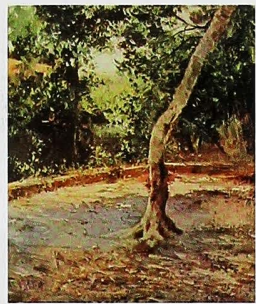

Fig.14 René Palma, Estudio de paisaje, (1908), (Variedades, Lima, No 447, 23 IX 1916).

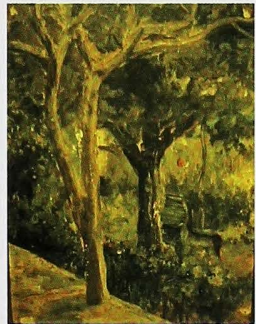

Fig. 15 Mercedes Dammert, Jardines (1906), óleo sobre lienzo, Lima, colección particular.

trabajos de sus discípulas Estío en la Quinta Heeren de María Angélica Quincot, Primeras rosas de Carmen Solari, Estudio de paisaje de René Palma (Fig.14) y Jardines de Mercedes Dammert 


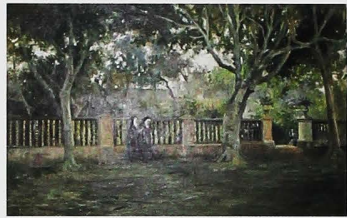

Fig.16 María Angélica Quincot, Parque antiguo, (1907). óleo sobre lienzo, colección particular.

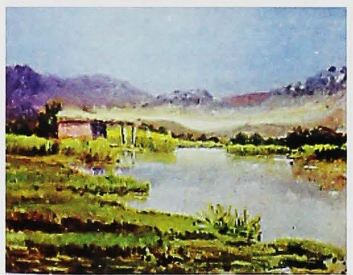

Fig. 17 Manuel Pardo y Heeren, Paisaje de villa (cercanías chorrillanas) (Variedades, Lima, No 431,3 VI 1916)
(Fig.15), composiciones que presentan plantas en primer plano, en su mayoría troncos de árboles.

Entre las creaciones puntuales que evidencian el interés del taller por la pintura al aire libre, se encuentran dos paisajes realizados por Quincot. El primero, Parque Antiguo. Quinta Heeren (1907), representa a dos religiosas -que apenas se advierten-en el patio, caminando alrededor del balcón y rodeadas de árboles. Un detalle que singulariza este cuadro es que constituye la primera pintura en la historia del arte peruano que incluye la representación de la propia artista haciendo su retrato $^{15}$. La pintora buscó enfatizar el ejercicio de su quehacer artístico al autorretratarse en la obra (Fig.16) ${ }^{16}$ en un segundo plano, junto a la puerta de entrada a los jardines. Otro alumno del taller fue Manuel Pardo Heeren, de 14 años, hijo del presidente Manuel Pardo (Castillo, 1919d:684). Este joven artista inició su labor con una obra pintada del natural $^{17}$ (Castillo, 1916b:691-692), Paisaje de villa (cercanías chorrillanas) (Fig.17), trabajo que Castillo publicó en el suplemento artístico de Variedades, donde ejercía la crítica de arte $^{18}$.

Los estudios del taller de Heeren continuaron en 1917 en el Centro Social de Bellas Artes. Los encargados de dictar los cursos eran Líbero Valente y Castillo, siendo este último responsable de impartir las clases de pintura. Además de los estudios de modelo vivo hechos en el local del centro, se organizaron excursiones semanales a los alrededores de la ciudad para pintar paisajes D'aprés nature ("De arte Nacional", 1917:2). Este nuevo lugar funcionó en el estudio del escultor Francisco Jáuregui, antiguo discípulo del taller de pintura de la Quinta Heeren, ubicado en la Plaza Bolívar. Una de las innovaciones en el estudio fue dictar las primeras clases de desnudo ("De arte Nacional", 1917:2). A pesar de tener en principio un carácter mixto, como se ve en el anuncio publicado en La Prensa ("Centro Social de Bellas Artes", 1917:5), es poco probable que haya tenido participación femenina.

El tipo de naturalismo que caracterizó a Castillo y a sus alumnos se basó en copiar del natural los objetos. Se preocupó por la incidencia de la luz y prefería encuadres que recuerdan a las estampas japonesas. Sin embargo, se diferencia del Impresionismo francés en los aspectos formales: en los cuadros de Monet y sus compañeros, el uso de los colores complementarios está en toda la obra, dispuestos de manera uniforme y vinculando fondos y figuras. Es un hecho conocido que los impresionistas necesitaron de la tranquilidad del

15 Para el caso de las pintoras españolas y sus retratos o autorretratos, ver: (De Diego, 1987:205 y 217).

16 Éste no es un hecho aislado: el propio maestro Castillo, en su época de formación, se había pintado en su paisaje Mañana frente al Huascaran (1881).

17 Castillo no sólo lo pondría de ejemplo, sino que lo mencionaría como un alumno destacado dentro de la academia de pintura de Heeren (Castillo, 1919d:684) y como un digno representante del taller (Castillo, 1920:518-520).

18 Este lugar, conocido actualmente como los Pantanos de Villa, definió el paradigma del tipo de paisajes que se ensenaba en la academia de pintura. 
estudio para terminar sus obras, y por tanto sus pinturas no son estrictamente del natural. El uso del color puro y de los complementarios es una característica común a todo el grupo; según dijo Monet en una entrevista de 1888: colour owes its brightness to force of contrast rather than to its inherent qualities;... primary colours look brightest when they are brought into contrast with their complementaries (Bonford et al., 1990:88).

Esta unidad técnica se rompe en la propuesta de Castillo y sus discípulos: algunas partes de sus obras están perfectamente acabadas, mientras que otras apenas se abocetan con unos trazos muy empastados. Es significativo que todos los cuadros representan a Lima, ciudad de nubosidad constante y dotada de una luz blanquecina. Las obvias diferencias con la luz brillante captada en Francia por los impresionistas o con la del Mediterráneo que podemos apreciar en las obras de los pintores españoles Mariano Fortuny o Joaquín Sorolla condicionaron el diferente resultado de las obras. Posteriormente el propio pintor definió el tipo de impresionismo del que decía formar parte en la polémica suscitada en torno al paisaje en 1916.

\section{La cerámica del Perú Antiguo en el taller de pintura}

Tengo fanatismo por la antigua cerámica nacional, pese a la desgracia que ella me ocasionará, sirviéndome de cebo para cometer la bobería supina de abandonar una posición hecha en la Argentina y venir aquí para casi atragantarme de miserias (Castillo, 1919b:524-528). Cuando el pintor escribía estas palabras, habían pasado ya trece años desde el inicio de la actividad del taIler de pintura de la Quinta Heeren; sin embargo, este desencanto ante un contexto local adverso nos revela el alta estima en que Castillo tenía a la cerámica del Perú antiguo.

El interés de Castillo por los objetos precolombinos está documentado antes que el de su alumna Elena Izcue, considerada como la pionera en rescatar la tradición artística de las formas del Perú Antiguo en el siglo XX (Wuffarden y Majluf, 1999:27). Hoy se conoce que el pintor escribió y realizó ilustraciones sobre el tema en 1912 que fueron publicadas en Ilustración Peruana (Villegas, 2006:119), aunque ya el taller de la Quinta Heeren puso de manifiesto su interés por la cerámica antigua, entusiasmo que inculcó a sus discípulas. El maestro incorporó los objetos precolombinos como modelos para el dibujo del natural, que aparecen en las obras presentadas por María Flórez de Quintanilla y Carmen Solari en la primera muestra del taller (1906). La primera presentó una composición a la manera de un bodegón Ilamada Pre-historia (Fig.18), cuya singularidad radicaba en la representación de dos ceramios sobre fondo neutro, uno con asa de estribo, propio de la cultura moche. Solari presentó Geranios y huacos, pero se desconoce su forma ya que no apareció publicada en Actualidades.

Al igual que los objetos cotidianos, los ceramios eran vistos como figuras decorativas y más como un recurso compositivo que como contenido programático.

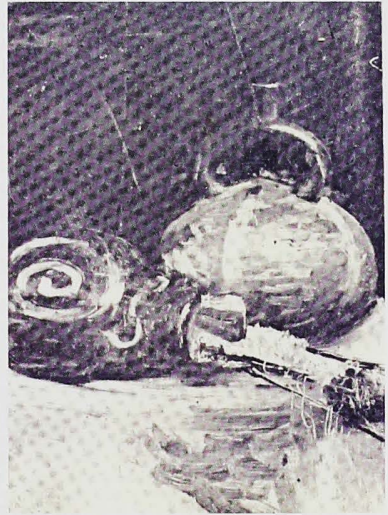

Fig.18 María Flores Quintanilla, Pre-historia (Actualidades, Lima, No 195,25 XII 1906, p.1368). 
Como tema, estuvo ausente en la segunda exposición realizada por los alumnos del taller en 1907. En el siglo XIX, Francisco Laso habia pintado en El habitante de las Cordilleras (1855) un personaje con vestimenta andina y que sostiene en sus manos un ceramio moche; lo mismo había ocurrido con Teófilo Castillo en su Manchay Puito (1886), donde colocó un huaco negro según refirió la prensa (Villegas, 2006:120). A diferencia de sus antecesores donde el ceramio era parte de una composición mayor, Flórez Quintanilla lo pintó de manera individualizada. Esto revela el interés del maestro por inculcar en los alumnos a valorar un pasado muchas veces denostado y poco conocido en el período. De esta forma, el arte del Perú antiguo ya era parte del aprendizaje del dibujo al natural' ${ }^{19}$.

A diferencia del arte moderno representado por Pablo Picasso, que desarrolló el Cubismo a partir de la interpretación de las piezas de África y Oceanía vistas en el Museo del Trocadero de París ${ }^{20}$. En la mirada de los artistas peruanos de las dos primeras décadas del siglo XX, la representación del ceramio es realista y responde a la copia del objeto en su forma literal. En la propuesta nacida en la Quinta Heeren y continuada por la pintora Elena Izcue, el ceramio del Perú Antiguo es visto como objeto calcográfico en lugar de sustentar un análisis interpretativo desde su propio lenguaje formal. Como parte de un occidente periférico, los intelectuales y artistas peruanos estaban demasiado preocupados por legitimar su parte occidental de raíz española. El objeto precolombino, principalmente la cerámica, acababa de ganarse su entrada en el ámbito del arte, pero todavía era un hecho muy reciente para que los pintores peruanos los estudiaran en profundidad o propusieran obras artísticas basadas en su legado.

\section{El fin del taller de pintura de la Quinta Heeren}

¿Qué propició que el taller no continuara con sus clases y éstas se suspendieran en 1916 ? Un factor a tener en cuenta fue lo ocurrido en el Concurso de pintura Concha de 1914, cuando el jurado decidió sortear el primer lugar que se disputaban Juanita Martínez La Torre, Carmen Solari de Balleuri y María Angélica Quincot, sorteo del que salió vencedora la primera. Este hecho motivó una carta de Castillo en la que anunciaba que sus alumnos no participarían en los siguientes concursos (Castillo, 1914b:6). Con ello se cerraba toda posibilidad de hacer visibles las obras producidas por las pintoras en el único certamen que se celebraba en Lima en ese momento.

La polémica se suscitó alrededor de los cuadros presentados por Carmen Solari de Ballauri ${ }^{21}$ y María Angélica Quincot, ambas alumnas del Taller de Pintura de la Quinta Heeren, la última sobrina de Daniel Castillo, hermano del maestro del taller ${ }^{22}$. Quincot tuvo una destacada participación en las muestras que se realizaron en el taller de pintura en 1906 y $1907^{23}$ y quedó como finalista en el Concurso de pintura Concha celebrado en 1914.

De un lado Quincot presentó obras donde destacaba el simbolismo con Ensoñación (1907) el naturalismo con Parque Antiguo. Quinta Heeren (1907) y la temática religiosa con Presentación

19 La valoración de los objetos precolombinos inculcado en el taller de pintura de Heeren dio sus frutos en su alumna Elena Izcue, que realizó el Arte Peruano en la Escuela (1925), publicado en París, que constituyó el primer manual infantil de dibujo a la acuarela y tinta inspirado en la gráfica de las culturas precolombinas. La pintora lo incorporó en la enseñanza para niños y más tarde en sus diseños de telas de alta costura.

20 De acuerdo con Clifford, Picasso llevó a cabo una apropiación del objeto con una relegación de lo primitivo a un pasado que se desvanece y un presente ahistorico y conceptual (Clifford, 1995:240).

21 Carmen Solari de Ballauri presentó nueve obras aunque no se conoce los títulos de ellas, nos quedan las referencias en la prensa que las describen como: "dos acuarelas de estilo arabesco", "La cabeza de un pescador chorrillano", "El malecón de Chorrillos", "Niña sentada en una pérgola" (Pachas, 2006:88-89).

22 Testimonio de ello es el retrato que Castillo hace a su cuñada, Adela Quincot de Castillo, que se conserva actualmente en el Museo de Arte de Lima. Ello pone en evidencia que el taller era un entorno familiar y reducido, circunscrito a un sector intelectual que compartía afición por el estudio de las artes.

23 En 1906 participa con nueve obras, ver:(Catálogo de la primera.... 1906). Al año siguiente, exhibe diez obras. ver: (Catálogo de la segunda.... 1907). 
de la virgen de Lourdes (1914), este último tema ausente en lo enseñado en el taller. La propuesta simbolista y la temática religiosa diferenciaban la propuesta de Quincot de la de su maestro. No obstante la crítica valoró negativamente algunas de sus obras, calificándolas de simples reproducciones de trabajos de Castillo. Por otro lado Juanita Martínez La Torre, la diletante auspiciada por Mariátegui, presentó Paisaje (Fig. 19) y Retrato de niño en donde a través de la crítica se conoce que eran copias. Esta forma de realizar una pintura contradecía los postulados naturalistas auspiciado por Castillo y sus discípulas en el taller de pintura y creemos fue la principal confrontación para negarse aceptar el fallo del jurado.

Pero quien empeoró la situación fue quizás José Carlos Mariáteguii ${ }^{24}$, al alegar la intervención del maestro en los cuadros de Quincot: sólo que en unos u otros se advierte a ratos para el ojo del observador la mano del maestro-maestro asequible y bondadoso-que dio algunos retoques delatores (Portocarrero, 1999:386). Las pintoras mujeres podían ser aceptadas siempre y cuando sus obras fueran producto de "aficionadas". Es decir podían ser artísticas pero no artistas (De Diego, 1986:356). Quincot tuvo que afrontar una censura que cuestionaba su capacidad creativa al vincular su producción como imitación de la de su maestro.

Una característica común entre los críticos del siglo XIX fue la de relacionar las obras de las artistas con la ayuda del maestro, del esposo o del padre, pues de esta manera se creía avalar a la pintora. Quienes han estudiado la trayectoria de las alumnas del taller no han dejado de mencionar las similitudes técnicas de sus obras con las del maestro ${ }^{25}$. Este es uno de los

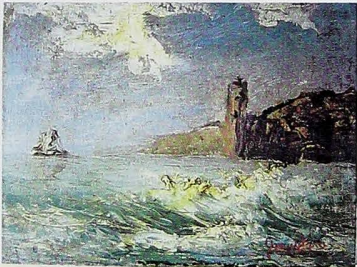

Fig. 19 Juanita Martínez La Torre, Paisaje (sin título), (c. 1914), óleo sobre madera, colección particular.

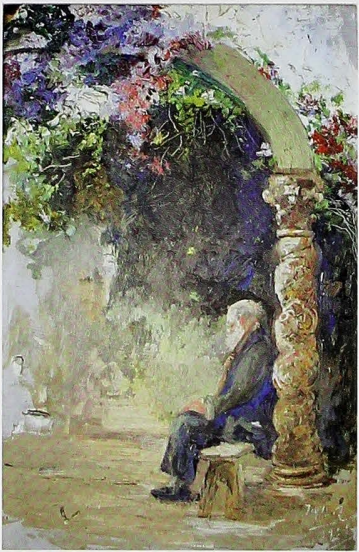

Fig.20 María Angélica Quincot, Anciano en reposo (c.1912. 14), óleo sobre lienzo, Lima, colección particular. hechos más reiterados y tristes a la hora de hablar de las pintoras, algo que hemos mencionado con anterioridad; pensar siempre que un hombre (padre, marido o maestro) ha participado en la elaboración de su obra (De Diego, 1987:253). Una pintura de Quincot que muestra la influencia de Castillo es Anciano en reposo (Fig. 20), con referencias, tanto compositivas como técnicas, a un óleo de Castillo, un estudio del natural tomado por el pintor en un asilo de ancianos y colocado como portada en Ilustración Peruana en 1912: Un rincón poético de Lima-El de los olvidados, en la hermanita de los pobres (Fig. 21). La columna salomónica es el elemento de apoyo que toma

24 Su objetivo era ayudar a una de las participantes, la diletante y joven pintora Juanita Martínez La Torre.

25 Por ejemplo, en la obra temprana de Elena Izcue se observa la influencia del maestro en el tratamiento de los jardines (Majluf y Wuffarden, 1999:34-35). Igualmente, se sitúa las obras de las discípulas dentro del estilo del maestro Castillo (Pachas, 2008:44). 


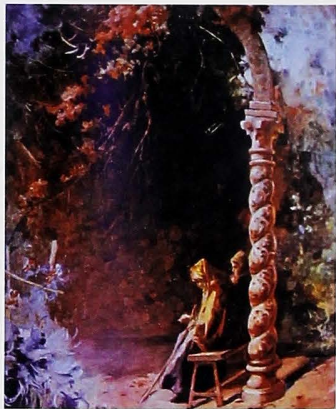

Fig.21 Teófilo Castillo, Un rincón poético de Lima - El de los olvidados, en la hermanita de los pobres (Ilustración Peruana, Lima, №141, 12 VI 1912).

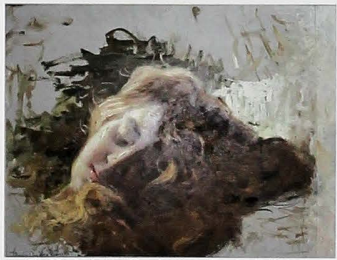

Fig.22 María Angélica Quincot, Ensoñación (1914). óleo sobre lienzo, Lima, colección particular.

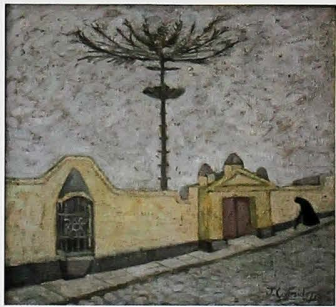

Fig.23 Julia Codesido, Convento de los Descalzos (1917), óleo sobre lienzo, colección particular. el artista para colocar dos ancianas sentadas a su costado. Quincot optó por representar a un hombre en el lado de la columna. La ejecución de la obra de la pintora revela la dependencia al maestro en la ejecución técnica, aunque las diferencias cromáticas reconocen la diferente autoría.

Sin embargo, Ensoñación (Fig.22), presentado por Quincot en el Concurso Concha de 1914, manifestó su independencia del maestro. Este retrato, de claros rasgos simbolistas, muestra la cabeza de una mujer dormida, con el cabello suelto. Castillo no fue favorable al Simbolismo ni lo trató en sus clases, sino que en su taller se fomentó el Naturalismo. No obstante, ya desde la segunda exhibición de 1907, varias de sus alumnas mostraron esta misma tipología representando un rostro femenino dormido e inserto en brumas ${ }^{26}$. En la obra de Mercedes Dammert se advierte una cercanía mayor a Castillo por su carácter conservador, ya que utiliza el mismo fondo verde que su maestro. El uso del blanco que hace Quincot para lograr efectos de luz en la frente del personaje se diferencia de la propuesta más académica de Dammert.

No todos los alumnos de Castillo manifestaron o se mantuvieron bajo la influencia técnica del maestro, sobre todo algunos que tenían experiencias previas en la práctica de la pintura. Entre la obra de Julia Codesido anterior a la Escuela Nacional de Bellas Artes (ENBA), y vinculada al taller de pintura mencionamos Convento de los Descalzos (Fig.23), fechada en 1917, quizá una de las primeras representaciones de un motivo que tiempo después tomó el pintor arequipeño Jorge Vinatea Reinoso en sus dos cuadros Descalzos, ambos de 1925. Esta obra muestra ya el afán sintetizador de la pintora en la ejecución de la mujer con manto negro y los elementos de arquitectura, a excepción de fondo del cielo, realizado de manera abocetada. La delimitación del color y la preferencia por la línea muestran que su concepción del arte es diferente a la de su maestro, Castillo. Así, se independiza su obra y se identifica un temperamento artístico singular. La pintora estudió en los centros europeos en

26 Lys d'or, de Margarita Buffet, y Give a rose, de Melania Araráz, mostraban esta misma propuesta. 
los que radicó y tenía una cultura artística temprana; por lo tanto, se trataba de una artista ya formada cuando empezó a asistir a las clases de Castillo. Pero éste no sería el único caso: la joven Clotilde Porras de Osma también tuvo una enseñanza previa con el pintor español radicado en el Perú, Ramón Muñiz, y en Madrid conoció a los pintores españoles Ignacio Zuloaga, Joaquín Sorolla y Manuel Benedito (Castillo, 1915:2923).

Pocas de las pintoras que estudiaron en Heeren convirtieron su afición en una ca-

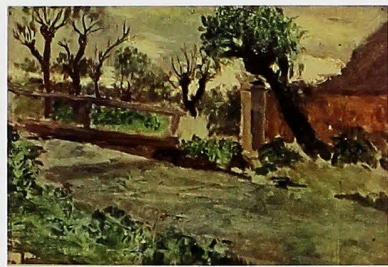

Fig.24.- Ricardo Flórez de Quintanilla, Después de la lluvia (Variedades, Lima, №435, 4 VII 1916) rrera. El primer deber de la mujer en el discurso ilustrado consistía en ser domésticas, en recluirse en círculo de la familia, abandonando otros espacios sociales y mundanos, para dedicarse totalmente a su esposo e hijos (Bolufer, 1998:273). Sin posibilidades de burlar el deber patriarcal de ser buenas esposas o buenas hijas $^{27}$, sólo les quedaba recurrir al diletantismo sin traspasar las fronteras de un estudio inicial, jamás profesional. En los límites espaciales del ámbito doméstico, su futuro era nulo para los estudios de la historia del arte.

Su educación se dirigía siempre al diletantismo en todos los campos y mucho más en el artístico, ya que la pintura era otro atractivo, el más refinado de los adornos. Pero lo verdaderamente trágico era que ese diletantismo sólo se podía desarrollar dentro de las estructuras familiares- la casa paterna y, posteriormente, la del marido (De Diego, 1987:215) ${ }^{28}$.

Estudiar en el taller de pintura fue una práctica común para las señoritas de sociedad, un atributo más para ser buenas esposas o para perfeccionar sus aptitudes artísticas. Por lo visto, en el taller de pintura de la Quinta Heeren fueron pocas las que traspasaron los límites que se les imponían para ejercer su oficio artístico. Estos fueron los casos de las pintoras Julia Codesido y Elena Izcue, los dos ejemplos más representativos de profesionalización de la vocación artística femenina ${ }^{29}$. Codesido, pintora destacada de la década de los veinte, ingresó en la Escuela Nacional de Bellas Artes (ENBA) y se vinculó inmediatamente al grupo dirigido por José Sabogal en su búsqueda de un arte propiamente peruano. Su carrera derivó progresivamente de un localismo vernáculo a la abstracción de sus obras de mediados de los cincuenta (Wuffarden, 2004). Izcue también estudió en la recién fundada ENBA. No sólo egresó con medalla de oro de su promoción, sino que intervino en el contexto local peruano de los años veinte, participó junto a sus profesores y compañeros del ENBA en la decoración del Salón Neo-peruano del Palacio de Gobierno, que conmemoraba el Centenario de la Batalla de Ayacucho (1924).

27 Este sería el caso de Mercedes Dammert, alumna en la Quinta Heeren cuyas obras se registran hasta su casamiento en 1910. Lo mismo ocurre con María Flórez de Quintanilla, la joven abandonó la carrera pictórica al contraer nupcias en 1912 con Guillermo Durand Fernández de Maldonado. En otro contexto, María Angélica Quincot tuvo que abandonar su vocación artística al encargarse del cuidado de su madre por ser hermana mayor:

28 Estrella de Diego refiere como la pintura y el dibujo son parte de la educación que toda señorita debía recibir durante el siglo XIX, ver: (De Diego, 1986:355).

29 Además de estas destacadas pintoras, se deben añadir los nombres de María Isabel Arenas y Loayza, René Palma y Carmen Elizalde, alumnas del Taller de Pintura de la Quinta Heeren que figuran matriculadas en la Escuela de Bellas Artes en sus primeros años (Monografia histórica..., 1922). 


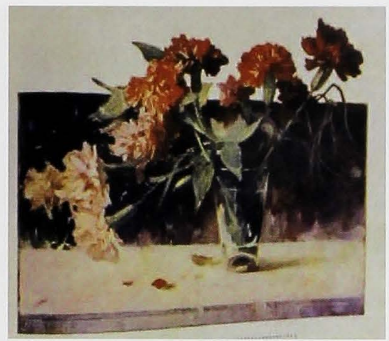

Fig.25 Teófilo Castillo, Claveles (Variedades. Lima, $N^{\circ} 412,22$ I 1916)

Otro alumno del taller de pintura fue Ricardo Flórez de Quintanilla ${ }^{30}$, alumno de Castillo, de quien dijo: (...) le conozco desde niño. Puedo decir que yo le puse los pinceles en la mano... Aseguro que constituye él un caso excepcional en nuestro ambiente pedestre (Castillo, 1919c:10). Flórez se convirtió en el más fiel seguidor de las enseñanzas impartidas en el taller, a través del estudio del natural y de la copia directa del paisaje (Fig.24). El maestro lo señaló como el primer pintor peruano en hacer Impresionismo, exceptuando sus propios intentos, que consideraba secundarios, y subrayó la sinceridad de su obra, desprovista de teologías o filosofias, sus árboles son eso, al igual que lo hicieron los italianos Sartorelli y Delleani (Castillo, 1916 c:832-833) 31. Flórez derivó en una técnica puntillista en su búsqueda de captar la luz.

Llama la atención que en 1916 el propio Castillo, director artístico de Variedades, publicara en la revista una tricromía de media docena de claveles en un vaso de cristal (Fig.25). El texto que acompañaba a la imagen decía que se trataba de una nota natural, sencilla y fácil, dirigida a las lectoras que quisieran pintar y supieran ver el color (Castillo, 1916a:121-122). Con ello el artista se mantenía en el proyecto de enseñanza que había iniciado en 1906 , que buscaba copiar de manera directa un modelo e iba dirigido a un público femenino ${ }^{32}$, que sería mayoría en su taller, como lo muestra Flor de Retama de Rosalía García de Lavalle y Pardo (Fig.26). La vocación por la enseñanza seguía presente en el maestro ${ }^{33}$, pero el paso siguiente era la Escuela Nacional de Bellas Artes (1919). A pesar de haber fomentado la materialización de esta institución a través de sus escritos, no fue llamado a integrar la plana docente. Su desengaño al no ingresar en la Escuela de Bellas Artes se puso de manifiesto en la siguiente declaración de Castillo:

...inútil toda mi labor de enseñanza del natural durante diez años en la Quinta Heeren y de la que fueron discípulos aventajados: Andrea Buffet, Julia Codesido, René Palma, Angélica Quincot, María Isabel Arenas, Mercedes Dammert, Luis Mora, Ricardo Flórez Quintanilla y Manuel Pardo Heeren. Todo al agua porque no supe hacer antesalas en palacio y he dado duro y seguiré dando á los pintamonas y los filisteos... (Castillo, 1919d:683-684).

En 1917, una nota escrita en La Prensa relacionada con la apertura de una Escuela de Dibujo Natural Vivo en Montevideo dio cuenta de la importancia de la obra de Castillo y su escuela: hay que hacerle, sin embargo, justicia al maestro Castillo, que fue el que inició el aprendizaje del natural rompiendo la tradición de las copias y dando por primera vez entre nosotros, toda su importancia al estudio de la luz ("Vida Artística Montevideana", 1917:2). Este es quizá el principal

30 Hermano de la alumna del taller, María Flórez de Quintanilla. Ambos eran hijos del médico Ricardo Flórez, célebre por introducir el primer automóvil en Lima. Eran sobrinos por vía materna del crítico de arte Emilio Gutiérrez de Quintanilla.

31 Antes que Flórez, habían exhibido en Lima su obra impresionista el catalán Oxandaberro y el chileno Lattanzi.

32 Estrella de Diego establece que los tratados de dibujo durante el siglo XIX estuvieron especialmente dirigidos a un público femenino, ver: (De Diego, 1986:358).

33 Sobre el interés de Teófilo Castillo por la enseñanza artística además del taller de Heeren, ver la nota 113 en: (Villegas, 2013:113). 
mérito de los estudios realizados en Heeren: la pintura al aire libre, el reconocimiento del entorno de la naturaleza, y los primeros inicios del Naturalismo, que en el Perú fue cultivado por la primera generación de pintoras mujeres, que, a diferencia de sus pares latinoamericanas o españolas, no limitaron su producción a retratos, bodegones o pintura de flores ${ }^{34}$, sino que su afán por captar el natural las llevó a trasladarse a las playas, a la sierra limeña y a los jardines del taller donde comenzó su educación artística ${ }^{35}$.

Existe una aparente contradicción entre la importancia de la enseñanza en Heeren y el diletantismo femenino, que provocó que la mayoría de las obras, por ser de aficionadas, se quedase en el interior de los hogares. Esta sería una característica del período para el desarrollo del arte moderno en el Perú el cual se gestó desde la considerada periferia artística, tanto en la práctica pictórica femenina vista como parte de los atributos de una señorita de sociedad. Este también fue

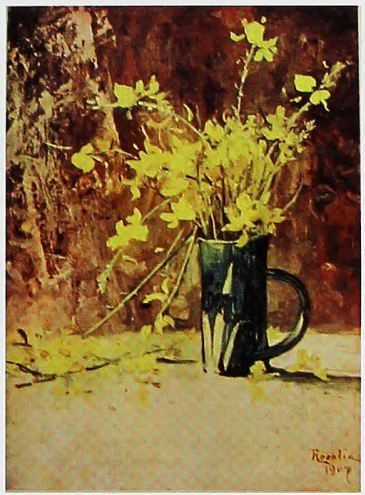

Fig.26 Rosalía García de Lavalle y Pardo, Flor de Retama (Variedades, Lima, No414, 5 II 1916) el caso de la incursión del arte moderno que tímidamente aparecía en la gráfica de las revistas ilustradas peruanas y las portadas de los libros de la primera década del XX. Debemos concluir que el taller de pintura de Heeren con sus pintoras mujeres fue la primera señal de modernidad en la plástica peruana local. Fue un espacio donde las pintoras no sólo se supeditaron al maestro sino que cada una diferenció sus propios lenguajes artísticos poniendo elementos simbolistas en sus retratos, abordando el tema religioso o esquematizando las imágenes.

34 En España, la participación de pintoras en las Exposiciones de Bellas Artes durante el siglo XIX abarcó los siguientes temas: naturalezas muertas, paisajes y figuras humanas (De Diego, 1987). Entre las pintoras latinoamericanas los temas preferidos fueron los retratos, las escenas de género y las naturalezas muertas (Pachas, 2008:25).

35 Hasta la fecha no existe ninguna documentación que avale la existencia de estudios tomados del natural por parte de las pintoras latinoamericanas o españolas. En México la escuela de pintura al aire libre fue resultado de una reacción contra la Academia, Alfredo Ramos Martínez fundó la primera escuela conocida como Barbizon en 1913 (Gutiérrez Viñuales, 1997:206). 


\section{Bibliografía}

Anónimo

1903 "Concurso Concha", Actualidades № 2 Lima, enero 10, pp.20-22.

1906 "El arte en Lima una academia de pintura", Actualidades, №176, Lima agosto 11 , pp.828-830.

1906 "Salón Castillo. La fiesta del Vernissage", Actualidades, № 196, Lima, diciembre 31 , p.1396.

1917 "Vida artística montevideana", La Prensa, Lima, abril 24, p.2.

1917 "De arte nacional", La Prensa, Lima, junio 21, p. 2 .

1922? Monografía histórica y documentada sobre la Escuela Nacional de Bellas Artes desde su fundación hasta la segunda exposición oficial, Lima, Perú.

Bolufer Peruga, Mónica

1998 Mujeres e llustración. La construcción de la feminidad en la ilustración española. Valencia, Dipitació de València.

Bonford, David, Kirby, Jo y otros

1990 Art in the making Impressionism, Great Britain, National Gallery Publications.

\section{Castillo, Teófilo}

1914a "Interiores limeños IV, casa del Dr. José A. de Lavalle y Pardo", Variedades, No 353, Lima, diciembre 5, pp.1520-1524.

1914b "Concurso Concha. Después del fallo", La Crónica, Lima, diciembre 5, p.6.

1915 "Interiores limeños XVIII, casa del Dr. D. Felipe de Osma y Pardo", Variedades, № 405, Lima, diciembre 4, pp.2920-2923.

1916a "Notas de arte. En el taller del fotógrafo Goyzueta", Variedades, №.412, enero 22, pp.121-122.

$1916 b$ "Notas de arte", Variedades, №.405, Lima, mayo 27, No 430, pp.691-692.

1916 c "Notas de arte", Variedades, №.435, Lima, julio 1, pp.832-833

1916d "Interiores limeños XXI, casa de la señorita María Isabel Sánchez Concha", Variedades, № 448, Lima, noviembre 30 , pp. $1279-1280$.

1918 "De arte", Variedades, № 557, Lima, noviembre 2, pp.1053-1055.

1919a "Una visita a la Escuela de Bellas Artes, las obras de Hernández", Variedades, $\mathrm{N}^{\circ}$ 582, Lima, abril 26, pp.345-347.

1919b "Vida limeña Tello en la universidad", Variedades, $\mathrm{N}^{\circ} 591$, Lima, junio 28. pp.524-528.

1919c "Semblanzas de Artistas VI. Ricardo Florez", Familia, № 5, Lima, junio, pp. 10-12.

1919d "De actualidad dos cartas de Enrique Barreda y Teófilo Castillo", Variedades, № 598, Lima, agosto 16, pp. 683-684.

1919e "Exposición escolar", Variedades, № 613 , Lima, octubre 29, pp.1010-1012.
1920 "De arte crítica retrospectiva-el caso Koek Koek-Mi despedida", Variedades, № 638, Lima, mayo 22, pp.518-520.

1906 Catálogo primera exposición de pintura de los discípulos del Sr. T. Castillo, Lima, Casa Courret (ATC).

1907 Catálogo segunda exposición de pintura de los discípulos del Sr. T. Castillo, Lima, Casa Courret (ATC).

1917 "Centro Social de Bellas Artes", $L a$ Prensa, Lima, marzo 23, p.5.

Cliford, James

1995 "Historias de los tribal y lo moderno", Dilemas de la Cultura, Barcelona, Gedisa, pp.229-256.

Conway Jill. K., Bourque, Susan C. y Scott, Joan W.

1997 "El concepto de Género", En: El Género: La construcción cultural de la diferencia sexual, México, Programa universitario de Estudios de Género de la UNAM, pp.7-11.

\section{De Diego, Estrella}

1987 La mujer y la pintura del XIX español (cuatrocientas olvidadas y algunas más), Madrid, Ediciones Cátedra, S.A.

1986 "Aprender a dibujar sin maestro. Los tratados de pintura del XIX y la educación femenina", Goya, №192, Mayo-Junio, Madrid, pp.355-361.

Denegri, Francesca

2004 El Abanico y la cigarrera. La primera generación de mujeres ilustradas en el Perú, Lima, Instituto de Estudios Peruanos.

\section{García, Elvira}

1925 La mujer peruana a través de los siglos. Lima, Imprenta Americana.

\section{Gutiérrez Viñuales, Rodrigo}

1997 Pintura, escultura y fotografía en Iberoamérica, siglos XIX y XX, Madrid, Ediciones Cátedra, S.A.

Majluf, Natalia y Wuffarden, Luis Eduardo

1999 Elena Izcue. El arte precolombino en la vida moderna, España, Museo de Arte de Lima y fundación Telefónica.

\section{Majluf, Natalia}

1993 "Entre pasatiempo y herramienta artesanal: Aspectos de la enseñanza del dibujo en el diecinueve (1)", Sequilao, $\mathrm{N}^{\circ}$ 3, Lima, pp.32-42. 
Pachas, Sofia

2005 Academia Concha (1890-1918), tesis para optar el título profesional de licenciada en arte, Universidad Nacional Mayor de San Marcos.

2006? Las artistas plásticas en Lima 1891-1918, Lima, Seminario de Historia Rural Andino-UNMSM, 2008.

\section{Portocarrero, Ricardo}

1999 "Sensualidad y estética en los escritos de Juan Croniqueur. (1914-1919)". Mujeres y Género en la Historia del Perú. Lima, CENDOC-Mujer, pp.373-393.

\section{Tickner, Lisa}

2001 "Modernist Art History: The Challenge of Feminism" (1988), En: FeminismArt-Theory. An Anthology 1968-2000, Singapure, Backwell Publishing, pp. 250-257.
Villegas, Fernando

2006 El Perú a través de la pintura y crítica de Teófilo Castillo, nacionalismo, modernización y nostalgia en la Lima del 900, Lima, Asamblea Nacional de Rectores.

2013 Vínculos artísticos entre España y Perú (1892-1929). Elementos para la construcción del imaginario nacional peruano, Madrid, Universidad Complutense de Madrid, Tesis doctoral en Historia del Arte.

\section{Wuffarden, Luis Eduardo}

2004 "Julia Codesido, entre el indigenismo y el espíritu de la modernidad". Julia Codesido (1883-1979) muestra antológica. Lima, Graficas Biblos S.A. 
